\title{
Lebesgue regularity for differential difference equations with fractional damping.
}

\author{
Claudio Leal \\ Universidad de Santiago de Chile, Facultad de Ciencias, Departamento de Matemática y Ciencia de la Computación, Las \\ Sophoras 173. Estación Central. Santiago.Chile.e-mail:claudio.leal@usach.cl \\ Carlos Lizama \\ Universidad de Santiago de Chile, Facultad de Ciencias, Departamento de Matemática y Ciencia de la Computación, Las \\ Sophoras 173. Estación Central. Santiago. Chile. e-mail: carlos.lizama@usach.cl \\ Marina Murillo-Arcila \\ Institut de Matemàtiques i Aplicacions de Castelló (IMAC), Universitat Jaume I, Campus del Riu Sec s/n, 12071 Castelló, \\ Spain.e-mail: murillom@uji.es
}

\begin{abstract}
Let $X$ be a Banach space. We provide necessary and sufficient conditions for existence and uniqueness of solutions belonging to the vector-valued space of sequences $\ell_{p}(\mathbb{Z}, X)$ for the linearized part of equations that can be modeled in the form

$$
\Delta^{\alpha} u(n)+\mu \Delta^{\beta} u(n)=A u(n)+G(u)(n)+f(n), \quad n \in \mathbb{Z}, \quad \alpha, \beta>0, \quad \mu \geq 0,
$$

where $f \in \ell_{p}(\mathbb{Z}, X), A$ is a closed linear operator with domain $D(A)$ defined on $X$ and $G$ is a nonlinear term. The operator $\Delta^{\gamma}$ denotes the fractional difference operator of order $\gamma>0$ in the sense of Grünwald-Letnikov. Our class of models includes the discrete time Klein-Gordon, telegraph and Basset equations, among others differential difference equations of interest. We prove a simple criteria that show existence of solutions assuming that $f$ is a small and that $G$ is at less of quadratic order.
\end{abstract}

\section{Introduction}

This paper is concerned with a wide class of mixed evolution equations that can be considered either as models for partial differential equations that are continuous in space but discrete in time [12], or systems of difference equations [4, 19, Chapter 3]. An additional feature of the class of models that we will study is that they admit the possibility of two fractional orders in the discrete variable.

Typical models that are included in this article corresponds to the discrete time Klein-Gordon equation

$$
\Delta u(n, x):=u(n+1, x)-u(n, x)=u_{x x}(n, x)-b u(n, x)+|u(n, x)|^{c} u(n, x), \quad n \in \mathbb{Z}, \quad x \in \Omega \subset \mathbb{R}^{N}
$$

and the discrete time telegraph equation

$$
\tau \Delta^{2} u(n, x)+\Delta u(n, x)=\rho u_{x x}(n, x), \quad n \in \mathbb{Z}, \quad \tau \geq 0, \rho>0, \quad x \in J \subset \mathbb{R},
$$

as well as fractional versions of them $[7,21]$. The discrete version of the Basset equation $[32,10]$

$$
\Delta^{2} u(n)+\mu \Delta^{3 / 2} u(n)+b u(n)=f(n), \quad n \in \mathbb{Z}, \quad \mu, b>0,
$$

will be also included in our framework. The study of the above equations on $\mathbb{Z}$ is suggested in order to investigate the uniqueness and causality of $p$-summable solutions [11]. More precisely, given a Banach space $X$, we ask ourselves for the following problem: Is it possible to characterize solely in terms of the data of a given mixed 
evolution equation, the existence and uniqueness of solutions that belong to the vector-valued space of sequences $\ell_{p}(\mathbb{Z} ; X) ?$.

We have success in solve this open problem for the following abstract model

$$
\Delta^{\alpha} u(n)+\mu \Delta^{\beta} u(n)=A u(n)+f(n), \quad n \in \mathbb{Z}, \quad \alpha, \beta>0, \quad \mu \geq 0,
$$

where $f \in \ell_{p}(\mathbb{Z}, X), A$ is a closed linear operator with domain $D(A)$ defined on $X$ and $\Delta^{\gamma}$ denotes the fractional difference operator of order $\gamma>0$ as defined recently in [1]. Roughly speaking, it corresponds to a slight variant of the Grünwald-Letnikov derivative. Compare Definition 2.3 below with [33, Section 3.3 formula (27)]. It is worthwhile to observe that, for instance, the model (1.1) includes the Basset equation (1.3) taking $X=\mathbb{C}, A=b I, \alpha=2$ and $\beta=3 / 2$ whereas it also includes the linear part of the Klein-Gordon equation (1.1) choosing $X=L^{2}(\Omega), A=\partial_{x x}-b, \alpha=1$ and $\mu=0$.

The modeling with fractional difference equations is a recent and promising area of research that has been developed from different sides of interest. For instance, Atici and Sengul [8] develop some basics results of discrete fractional calculus. This authors introduce and solve Gompertz fractional difference equation for tumor growth models. See also [9] for related results in this direction. The methodology used in such discrete fractional calculus was extended in [29] to the context of abstract models, including in this way the handling of difference differential equations by methods of functional analysis and operator theory. Studies on qualitative properties, as for example the existence of positive solutions for discrete fractional systems, have been provided by Goodrich [14, 22, 23]. Other contributions are due to Ferreira [20], Holm [24], Kovács, Li and Lubich [27], Dassios [15, 17], Baleanu [16] and Tarasov [34].

Starting with the work of S. Blunck [13], existence and uniqueness of solutions for discrete systems that have the property of belong to the Lebesgue space of vector-valued sequences has been considered by many authors $[25,26,28,30]$ and some of them from a numerical point of view [27]. However, none of them have considered causal solutions, i.e. solutions with domain on $\mathbb{Z}$. On the other hand, some abstract models that are less general than ours have been recently reported in the literature and analyzed from diverse perspectives. For instance, it is shown in [1], that the semilinear problem

$$
\Delta^{\alpha} u(n)=A u(n+1)+f(n, u(n)), \quad n \in \mathbb{Z}, \quad 0<\alpha \leq 1,
$$

admits almost automorphic solutions whenever the operator $A$ is the generator of a $C_{0}$-semigroup and $f$ satisfies Lipschitz conditions of global and local type. We note that in such paper an application to a model of population of cells is also given. Existence of weighted pseudo almost automorphic solutions to (1.5) have been also investigated in [6].

This paper is organized as follows: In Section 2, we first recall the notions of $U M D$-spaces, $R$-boundedness and the discrete time Fourier transform defined over the space of distributions. These concepts allow us to formulate Blunck's Fourier multiplier theorem for operator-valued symbols on $U M D$-spaces [13]. In Section 3, we prove our main result, that is, if

$$
\left\{\left(1-e^{-i t}\right)^{\alpha}+\mu\left(1-e^{-i t}\right)^{\beta}\right\}_{t \in \mathbb{T}} \subset \rho(A), \quad \mu \geq 0, \quad \alpha, \beta>0, \quad \mathbb{T}:=(-\pi, \pi),
$$

where $\rho(A)$ denotes the resolvent set of $A$, then the following assertions are equivalent:

i) For all $f \in \ell_{p}(\mathbb{Z}, X)$ the problem $\Delta^{\alpha} u(n)+\mu \Delta^{\beta} u(n)=A u(n)+f(n), n \in \mathbb{Z}$, has a unique solution in $\ell_{p}(\mathbb{Z},[D(A)])$

ii) $M(t):=\left(\left(1-e^{-i t}\right)^{\alpha}+\mu\left(1-e^{-i t}\right)^{\beta}-A\right)^{-1}$ is an $\ell_{p}$-multiplier from $X$ to $[D(A)]$;

iii) The set $\{M(t)\}_{t \in \mathbb{T}}$ is $R$-bounded.

Furthermore in the context of Hilbert spaces a simpler criteria is also provided, replacing the condition (iii) above by

$$
\sup _{t \in \mathbb{T}}\|M(t)\|<\infty .
$$

As a consequence, we analyze the nonlinear equation

$$
\Delta^{\alpha} u(n)+\mu \Delta^{\beta} u(n)=A u(n)+G(u)(n)+g(n), \quad n \in \mathbb{Z},
$$


where $g \in \ell_{p}(\mathbb{Z}, X)$ and $G: \ell_{p}(\mathbb{Z}, X) \rightarrow \ell_{p}(\mathbb{Z}, X)$ are given. We show that if $G(0)=G^{\prime}(0)=0$ and $g$ is small enough, then the nonlinear equation has at least one solution in $\ell_{p}(\mathbb{Z}, X)$. Finally, in section 4 , we show, as an application of our characterization, that for all $0<\alpha, \beta<2$ and $b>2^{\alpha}+\mu 2^{\beta}$ we can find $\varepsilon^{*}>0$ such that for all $\varepsilon \in\left(0, \varepsilon^{*}\right)$, there exists $u^{\epsilon} \in \ell_{p}\left(\mathbb{Z}, L^{2}(\mathbb{R})\right)$ that solves the problem

$$
\Delta^{\alpha} u^{\epsilon}(n, x)+\mu \Delta^{\beta} u^{\epsilon}(n, x)=u_{x x}^{\epsilon}(n, x)-b u^{\epsilon}(n, x)+\left|u^{\epsilon}(n, x)\right|^{c} u^{\epsilon}(n, x)+\varepsilon f(n, x), n \in \mathbb{Z}, x \in \Omega \subset \mathbb{R}^{N},
$$

for any $c>1$.

\section{Preliminaries}

In this section we recall some concepts about fractional derivatives, the Discrete Time Fourier Transform, in short, DTFT, and operator-valued Fourier multipliers theorems defined on UMD spaces. For more details see $[5,18]$ and the references therein.

Definition 2.1. Let $X$ be a Banach space. $X$ is said to be a UMD space if it has the Unconditional Martingale Difference property (UMD), in other words, for each $p>1$, there exists a constant $C_{p}>0$ such that for any $\left(f_{n}\right)_{n \geq 0} \subset L^{p}(\Omega, \Sigma, \mu ; X)$ and any choice of signs $\left(\xi_{n}\right)_{n \geq 0} \subset(-1,1)$ and any $N \in \mathbb{Z}_{+}$we have the following estimate

$$
\left\|f_{0}+\sum_{n=1}^{N} \xi_{n}\left(f_{n}-f_{n-1}\right)\right\|_{L^{p}(\Omega, \Sigma, \mu ; X)} \leq C_{p}\left\|f_{N}\right\|_{L^{p}(\Omega, \Sigma, \mu ; X)} .
$$

In what follows, we denote by $\mathcal{B}(X, Y)$ the space of bounded linear operators between Banach spaces $X$ and $Y$ endowed with the uniform operator topology; when $X=Y$, we denote it by $\mathcal{B}(X)$.

Definition 2.2. Let $X$ and $Y$ be a Banach spaces. A subset of $\mathcal{T} \subset \mathcal{B}(X, Y)$ is called R-bounded if there is a constant $c \geq 0$ such that

$$
\left\|\left(T_{1} x_{1}, \ldots, T_{n} x_{n}\right)\right\|_{R} \leq c\left\|\left(x_{1}, \ldots, x_{n}\right)\right\|_{R}
$$

for all $T_{1}, \ldots, T_{n} \in \mathcal{T}, x_{1}, \ldots, x_{n} \in X, n \in \mathbb{N}$ where

$$
\left\|\left(x_{1}, \ldots, x_{n}\right)\right\|_{R}:=\frac{1}{2^{n}} \sum_{\varepsilon_{j} \in\{-1,1\}^{n}}\left\|\sum_{j=1}^{n} \varepsilon_{j} x_{j}\right\|
$$

for $x_{1}, \ldots, x_{n} \in X$.

Given $u \in \ell_{p}(\mathbb{Z} ; X)$ and $v \in \ell_{1}(\mathbb{Z})$ we define the convolution product

$$
(u * v)(n):=\sum_{j=-\infty}^{n} u(n-j) v(j)=\sum_{j=0}^{\infty} u(j) v(n-j), \quad n \in \mathbb{Z} .
$$

For any $\alpha \in \mathbb{R}$, we set

$$
k^{\alpha}(n)=\left\{\begin{array}{cc}
\frac{\alpha(\alpha+1) \ldots(\alpha+n-1)}{n !} & n \in \mathbb{N}_{0} \\
0 & \text { otherwise }
\end{array}\right.
$$

where $\Gamma$ is the Euler gamma function. Some properties related to the special kernel $k^{\alpha}$ can be found in $[2$, Section 2] and [29].

Definition 2.3. Let $\alpha>0$ be given and $f: \mathbb{Z} \rightarrow X$ a vector-valued sequence. We define the fractional sum of order $\alpha$ as follows

$$
\Delta^{-\alpha} f(n):=\left(k^{\alpha} * f\right)(n)=\sum_{j=-\infty}^{n} k^{\alpha}(n-j) f(j), \quad n \in \mathbb{Z}
$$

and the fractional difference of order $\alpha$ is defined by

$$
\Delta^{\alpha} f(n):=\left(k^{-\alpha} * f\right)(n)=\sum_{j=-\infty}^{n} k^{-\alpha}(n-j) f(j)=\sum_{j=0}^{\infty} k^{-\alpha}(j) f(n-j) .
$$


Given $\alpha \in \mathbb{R} \backslash\{-1,-2, .$.$\} , we have k^{\alpha}(n)=\frac{\Gamma(\alpha+n)}{\Gamma(\alpha) \Gamma(n+1)}$, where $\Gamma$ is the Euler function see [[33], formula (27), with $h=1$. The numbers $k^{\alpha}$ are known as Cesàro numbers of order $\alpha$. They were introduced by Zygmund in $[35$, p. 77] and rediscovered in several instances. The above definition of fractional difference operator of order $\alpha$ (for $n \in \mathbb{Z}$ ) was first introduced by Abadías and Lizama [1], after previous work of Lizama [29], as follows:

$$
W^{\alpha} f(n):=(-1)^{m} \Delta^{m} W^{-(m-\alpha)} f(n), \quad n \in \mathbb{Z},
$$

where $m:=[\alpha]+1$. In the above cited references it is also called Weil difference operator of order $\alpha$ and is denoted by $W$ instead of $\Delta$. Their equivalence with (2.3) was recently proved in [3, Theorem 2.3].

In what follows, we detail the definition and properties of the DTFT in the vector-valued Lebesgue space of sequences $\ell_{p}(\mathbb{Z}, X)$. We denote by $\mathcal{S}(\mathbb{Z} ; X)$ the space of all vector-valued sequences $f: \mathbb{Z} \rightarrow X$ such that for each $k \in \mathbb{N}_{0}$ there exists $C_{k}$ with

$$
p_{k}(f):=\sup _{n \in \mathbb{Z}}|n|^{k}\|f(n)\|<C_{k} .
$$

We also denote by $C_{p e r}^{n}(\mathbb{R} ; X), n \in \mathbb{N}_{0}$, the space of all $2 \pi$-periodic $X$-valued and $n$-times continuously differentiable functions defined in $\mathbb{R}$.

Let $\mathbb{T}:=(-\pi, \pi)$ and $\mathbb{T}_{0}:=(-\pi, \pi) \backslash\{0\}$. We introduce the space of test functions as $C_{p e r}^{\infty}(\mathbb{T} ; X):=$ $\bigcap_{n \in \mathbb{N}_{0}} C_{p e r}^{n}(\mathbb{R} ; X)$ endowed with the topology induced by the countable family of seminorms:

$$
q_{k}(\varphi)=\max _{k \in \mathbb{N}_{0}} \sup _{t \in[-\pi, \pi]}\left\|\varphi^{(k)}(t)\right\| .
$$

If $X=\mathbb{C}$ we simply denote $C_{\text {per }}^{\infty}(\mathbb{T} ; X)=C_{\text {per }}^{\infty}(\mathbb{T})$ and $\mathcal{S}(\mathbb{Z} ; X)=\mathcal{S}(\mathbb{Z})$.

We also consider the following spaces of vector-valued distributions

$$
\mathcal{S}^{\prime}(\mathbb{Z} ; X):=\{T: \mathcal{S}(\mathbb{Z}) \rightarrow X: T \text { is linear and continuous }\}
$$

and

$$
\mathcal{D}^{\prime}(\mathbb{T} ; X):=\left\{T: C_{p e r}^{\infty}(\mathbb{T}) \rightarrow X: T \text { is linear and continuous }\right\},
$$

equipped with the bounded convergence topology.

Remark 2.4. We can identify $\ell_{p}(\mathbb{Z} ; X)$ with a subspace of $\mathcal{S}^{\prime}(\mathbb{Z} ; X)$ via the mapping

$$
T_{f}(\psi):=\left\langle T_{f}, \psi\right\rangle:=\sum_{n \in \mathbb{Z}} f(n) \psi(n), \quad \psi \in \mathcal{S}(\mathbb{Z}),
$$

and we have $T_{f} \in \mathcal{S}^{\prime}(\mathbb{Z}, X)$. The space $C_{\text {per }}^{\infty}(\mathbb{T} ; X)$ can be also identified with a subspace of $\mathcal{D}^{\prime}(\mathbb{T} ; X)$ via the linear map

$$
L_{S}(\varphi):=\left\langle L_{S}, \varphi\right\rangle:=\frac{1}{2 \pi} \int_{-\pi}^{\pi} \varphi(t) S(t) d t, \quad \varphi \in C_{p e r}^{\infty}(\mathbb{T}),
$$

and we get $L_{S} \in \mathcal{D}^{\prime}(\mathbb{T} ; X)$.

We next recall the following definition.

Definition 2.5. The discrete time Fourier transform $\mathcal{F}: \mathcal{S}(\mathbb{Z} ; X) \rightarrow C_{p e r}^{\infty}(\mathbb{T} ; X)$ is given by

$$
\mathcal{F} \varphi(t) \equiv \widehat{\varphi}(t):=\sum_{j=-\infty}^{\infty} e^{-i j t} \varphi(j), \quad t \in(-\pi, \pi] .
$$

It is an isomorphism whose inverse is defined by

$$
\mathcal{F}^{-1} \varphi(n) \equiv \check{\varphi}(n):=\frac{1}{2 \pi} \int_{-\pi}^{\pi} \varphi(t) e^{i n t} d t, \quad n \in \mathbb{Z},
$$

where $\varphi \in C_{\text {per }}^{\infty}(\mathbb{T} ; X)$. 
Remark 2.6. This isomorphism, allows to define the discrete time Fourier transform (DTFT) between the spaces of distributions $\mathcal{S}^{\prime}(\mathbb{Z} ; X)$ and $\mathcal{D}^{\prime}(\mathbb{T} ; X)$ as follows

$$
\langle\mathcal{F} T, \psi\rangle \equiv \mathcal{F}(T)(\psi):=\widehat{T}(\psi) \equiv\langle T, \check{\psi}\rangle, \quad T \in \mathcal{S}^{\prime}(\mathbb{Z} ; X), \quad \psi \in C_{\text {per }}^{\infty}(\mathbb{T}),
$$

whose inverse $\mathcal{F}^{-1}: \mathcal{D}^{\prime}(\mathbb{T} ; X) \rightarrow \mathcal{S}^{\prime}(\mathbb{Z} ; X)$ is given by

$$
\left\langle\mathcal{F}^{-1} L, \psi\right\rangle \equiv \mathcal{F}^{-1}(L)(\psi):=\check{L}(\psi) \equiv\langle L, \widehat{\psi}\rangle, \quad L \in \mathcal{D}^{\prime}(\mathbb{T} ; X), \quad \psi \in \mathcal{S}(\mathbb{Z}) .
$$

In particular, we get

$$
\left\langle\mathcal{F} T_{f}, \varphi\right\rangle=\left\langle T_{f}, \check{\varphi}\right\rangle=\sum_{n \in \mathbb{Z}} f(n) \check{\varphi}(n), \quad \varphi \in C_{p e r}^{\infty}(\mathbb{T}), \quad f \in \ell_{p}(\mathbb{Z}, X) .
$$

Observe that the convolution of a distribution $T \in \mathcal{S}^{\prime}(\mathbb{Z}, X)$ with a function $a \in \ell_{1}(\mathbb{Z})$ verifies

$$
\langle T * a, \varphi\rangle:=\langle T, a \circ \varphi\rangle, \quad \varphi \in \mathcal{S}(\mathbb{Z}),
$$

where

$$
(a \circ \varphi)(n):=\sum_{j=0}^{\infty} a(j) \varphi(j+n) .
$$

From [29], the following generation formula holds

$$
\sum_{j=0}^{\infty} k^{\beta}(j) z^{j}=\frac{1}{(1-z)^{\beta}}, \quad \beta \in \mathbb{R}, \quad|z|<1,
$$

see also [35, p.42 formulae (1) and (8)]. In particular, for all $\alpha \in \mathbb{R}_{+}$we have that the radial limit exists and

$$
\widehat{k^{-\alpha}}(t)=\sum_{j=0}^{\infty} k^{-\alpha}(j) e^{-i t j}=\frac{1}{\left(1-e^{-i t}\right)^{-\alpha}}=\left(1-e^{-i t}\right)^{\alpha}, \quad t \in \mathbb{T}_{0} .
$$

Observe that $k^{-\alpha} \in \ell_{1}(\mathbb{Z})$ (see also [35, p.42 formula (2)]).

We also recall the following lemma stated in [31] which will be used in the proof of our main result.

Lemma 2.7. Let $u, v \in \ell_{p}(\mathbb{Z} ; X)$ and $a \in \ell_{1}(\mathbb{Z})$. The following assertions are equivalent:

i) $(a * u)(n)=v(n)$ for all $n \in \mathbb{Z}$.

ii) $\left\langle u, \check{\phi}>=<v,\left(\phi \cdot \widehat{a}_{-} \check{)}>\right.\right.$ for all $\phi \in C^{\infty}((-\pi, \pi), \mathbb{R})$, where

$$
\left(\phi \cdot \widehat{a}_{-} \check{)}(n):=\frac{1}{2 \pi} \int_{-\pi}^{\pi} \widehat{a}(-t) \phi(t) e^{i n t} d t, \quad n \in \mathbb{Z} .\right.
$$

We can now introduce the following notion of $\ell_{p}$-multiplier.

Definition 2.8. Let $X, Y$ be Banach spaces, $1<p<\infty$. A function $M \in C_{p e r}^{\infty}(\mathbb{T}, \mathcal{B}(X, Y))$ is an $\ell_{p}$-multiplier $\left(\right.$ from $X$ to $Y$ ) if there exists a bounded operator $T: \ell_{p}(\mathbb{Z} ; X) \rightarrow \ell_{p}(\mathbb{Z} ; Y)$ such that

$$
\sum_{n \in \mathbb{Z}}(T f)(n) \check{\varphi}(n)=\sum_{n \in \mathbb{Z}}\left(\varphi \cdot M_{-} \check{)}(n) f(n)\right.
$$

for all $f \in \ell_{p}(\mathbb{Z} ; X)$ and all $\varphi \in C_{p e r}^{\infty}(\mathbb{T})$. Here

$$
\left(\varphi \cdot M_{-} \check{)}(n):=\frac{1}{2 \pi} \int_{-\pi}^{\pi} e^{i n t} \varphi(t) M(-t) d t, \quad n \in \mathbb{Z} .\right.
$$


We finally recall the following Fourier multiplier theorem for operator valued symbols due to S. Blunck, see $[13,5]$ for more details. This theorem will be crucial for showing our main characterization. Blunck's theorem and its converse establishes an equivalence between $R$-bounded sets and $l_{p}$-multipliers.

Theorem 2.9. [13, Theorem 1.3] Let $p \in(1, \infty)$ and let $X, Y$ be $U M D$ spaces. Let $M \in C_{\text {per }}^{\infty}(\mathbb{T}, \mathcal{B}(X ; Y))$ such that the sets

$$
\left\{M(t),\left(1-e^{i t}\right)\left(1+e^{i t}\right) M^{\prime}(t): t \in \mathbb{T}_{0}\right\}
$$

are both $R$-bounded. Then $M$ is an $\ell_{p}$-multiplier (from $X$ to $Y$ ) for $1<p<\infty$.

The converse of Blunck's theorem holds without any restriction on the Banach spaces $X, Y$ in the following sense.

Theorem 2.10. [13, Proposition 1.4] Let $p \in(1, \infty)$ and let $X, Y$ be Banach spaces. Let $M: \mathbb{T} \rightarrow \mathcal{B}(X ; Y)$ be an operator valued function. Suppose that there is a bounded operator $T_{M}: l_{p}(\mathbb{Z} ; X) \rightarrow l_{p}(\mathbb{Z} ; Y)$ such that $(2.6)$ holds. Then the set

$$
\{M(t): t \in \mathbb{T}\}
$$

is $R$-bounded.

\section{A characterization of maximal regularity}

In this section, we first provide a characterization on the existence and uniqueness of solutions in $\ell_{p}(\mathbb{Z} ;[D(A)])$ for the general model

$$
\Delta^{\alpha} u(n)+\lambda \Delta^{\beta} u(n)=A u(n)+f(n),
$$

where $\alpha, \beta>0, \lambda \geq 0, A$ is a closed linear operator defined on a Banach space $X$ and $f: \mathbb{Z} \rightarrow X$ is a vectorvalued sequence. Recall that the above model is an abbreviated form to write a partial differential equation which is continuous in space but discrete in time. For example, the equation

$$
u(n+2, x)-2 u(n+1, x)+u(n, x)+\lambda[u(n+1, x)-u(n, x)]=\partial_{x x} u(n, x)+f(n, x), \quad x \in \Omega \subset \mathbb{R}^{N},
$$

fits in the abstract setting of the model (3.1) with $\alpha=2, \beta=1$ and $A=\partial_{x x}$.

We introduce the following definition, also called $\ell_{p}$-well-posedness in the literature.

Definition 3.1. Let $1<p<\infty$. We say that (3.1) has maximal $\ell_{p}$-regularity if for each $f \in \ell_{p}(\mathbb{Z} ; X)$ there exists a unique solution $u \in \ell_{p}(\mathbb{Z} ;[D(A)])$ of $(3.1)$.

We are ready to prove our main result:

Theorem 3.2. Let $A$ be a closed linear operator defined on an UMD space $X$. Set $\alpha, \beta>0$ and $\lambda \geq 0$. Suppose that

$$
\left\{\left(1-e^{-i t}\right)^{\alpha}+\lambda\left(1-e^{-i t}\right)^{\beta}\right\}_{t \in \mathbb{T}} \subset \rho(A)
$$

and define $M(t):=\left(\left(1-e^{-i t}\right)^{\alpha}+\lambda\left(1-e^{-i t}\right)^{\beta}-A\right)^{-1}$. Then the following assertions are equivalent

i) (3.1) has maximal $\ell_{p}$-regularity.

ii) $M(t)$ is an $\ell_{p}$-multiplier from $X$ to $[D(A)]$.

iii) $\{M(t)\}_{t \in \mathbb{T}}$ is $R$-bounded.

Proof. We first show $($ iii $) \Rightarrow(i i)$. Let $\{M(t): t \in \mathbb{T}\}$ be R-bounded. We will show that the set $\left\{\left(1-e^{i t}\right)(1+\right.$ $\left.\left.e^{i t}\right) M(t): t \in \mathbb{T}\right\}$ is also R-bounded. Defining for each $t \in \mathbb{T}, f_{\alpha}(t):=\left(1-e^{-i t}\right)^{\alpha}$ and $f_{\beta}(t):=\left(1-e^{-i t}\right)^{\beta}$ it can be shown that

$$
M^{\prime}(t)=-M(t)^{2}\left(f_{\alpha}^{\prime}(t)+\lambda f_{\beta}^{\prime}(t)\right)
$$

Since $f_{\alpha}^{\prime}(t)=i \alpha f_{\alpha}(t) \frac{1}{1-e^{i t}}$, it follows that $\left(1-e^{i t}\right)\left(1+e^{i t}\right) M^{\prime}(t)=i\left(\alpha f_{\alpha}(t)+\lambda \beta f_{\beta}(t)\right)\left(1+e^{i t}\right) M(t)^{2}$. From [5, Proposition 2.2.5] we deduce that the set $\left\{\left(1-e^{i t}\right)\left(1+e^{i t}\right) M^{\prime}(t): t \in \mathbb{T}\right\}$ is $R$ - bounded and the claim is proved. Consequently, by Theorem 2.9, we obtain $(i)$. 
The implication $($ ii $) \Longrightarrow($ iii $)$ follows immediatly from Theorem 2.10.

Let now show that $(i) \Longrightarrow(i i)$. Let $f \in \ell_{p}(\mathbb{Z}, X)$ be given. Then there exists a unique $u_{f} \in \ell_{p}(\mathbb{Z},[D(A)])$ solution of (3.1). We define $T_{\alpha, \beta}: \ell_{p}(\mathbb{Z}, X) \rightarrow \ell_{p}(\mathbb{Z},[D(A)])$ the linear operator given by $T_{\alpha, \beta}(f)=u_{f}$. By the Closed Graph Theorem, we get that $T_{\alpha, \beta}$ is bounded. Let $\varphi \in C_{p e r}^{\infty}(\mathbb{T}), f \in \ell_{p}(\mathbb{Z} ; X)$ and $u=T_{\alpha, \beta} f$. Since $k^{-\alpha} \in \ell_{1}(\mathbb{Z})$ we obtain the following identity,

$$
\begin{aligned}
\left(k^{-\alpha} \circ \check{S}\right)(n) & =\sum_{j=0}^{\infty} k^{-\alpha}(j) \check{S}(j+n)=\sum_{j=0}^{\infty} k^{-\alpha}(j) \frac{1}{2 \pi} \int_{-\pi}^{\pi} e^{i(n+j) t} S(t) d t \\
& =\frac{1}{2 \pi} \int_{-\pi}^{\pi} e^{i n t}\left(\sum_{j=0}^{\infty} e^{i j t} k^{-\alpha}(j)\right) S(t) d t \\
& =\frac{1}{2 \pi} \int_{-\pi}^{\pi} e^{i n t} \widehat{k}^{-\alpha}(-t) S(t) d t=\left(\widehat{k}_{-}^{-\alpha} \cdot S\right)(n),
\end{aligned}
$$

valid for any $S \in C_{\text {per }}^{\infty}(\mathbb{T}, \mathcal{B}(X, Y))$. Therefore, using the hypothesis and the observation that we have $M \in$ $C_{\text {per }}^{\infty}(\mathbb{T}, \mathcal{B}(X,[D(A)]))$ we get

$$
\begin{aligned}
\left\langle T_{\alpha, \beta} f, \check{\varphi}\right\rangle= & \langle u, \check{\varphi}\rangle=\sum_{n \in \mathbb{Z}} \check{\varphi}(n) u(n)=\sum_{n \in \mathbb{Z}} \frac{1}{2 \pi} \int_{-\pi}^{\pi} e^{i n t} \varphi(t) u(n) d t \\
& =\sum_{n \in \mathbb{Z}} \frac{1}{2 \pi} \int_{-\pi}^{\pi}\left(1-e^{i t}\right)^{\alpha} e^{i n t} \varphi(t)\left(\left(1-e^{i t}\right)^{\alpha}+\lambda\left(1-e^{-i t}\right)^{\beta}-A\right)^{-1} u(n) d t \\
& +\lambda \sum_{n \in \mathbb{Z}} \frac{1}{2 \pi} \int_{-\pi}^{\pi}\left(1-e^{i t}\right)^{\beta} e^{i n t} \varphi(t)\left(\left(1-e^{i t}\right)^{\alpha}+\lambda\left(1-e^{-i t}\right)^{\beta}-A\right)^{-1} u(n) d t \\
& -\sum_{n \in \mathbb{Z}} \frac{1}{2 \pi} \int_{-\pi}^{\pi}\left(\left(1-e^{i t}\right)^{\alpha}+\lambda\left(1-e^{-i t}\right)^{\beta}-A\right)^{-1} A u(n) e^{i n t} \varphi(t) d t \\
= & \sum_{n \in \mathbb{Z}} \frac{1}{2 \pi} \int_{-\pi}^{\pi} e^{i n t} \widehat{k}^{-\alpha}(-t) \varphi(t) M(-t) u(n) d t+\lambda \sum_{n \in \mathbb{Z}} \frac{1}{2 \pi} \int_{-\pi}^{\pi} e^{i n t} \widehat{k}^{-\beta}(-t) \varphi(t) M(-t) u(n) d t \\
& -\sum_{n \in \mathbb{Z}} \frac{1}{2 \pi} \int_{-\pi}^{\pi} e^{i n t} \varphi(t) M(-t) A u(n) d t \\
= & \left\langle u,\left(\left(k_{-}^{-\alpha}+\lambda k_{-}^{-\beta}\right) \cdot \varphi \cdot M_{-}\right)\right\rangle-\left\langle A u,\left(\varphi \cdot M_{-}\right)\right\rangle \\
= & \left\langle u,\left(k_{-}^{-\alpha}+\lambda k_{-}^{-\beta}\right) \circ\left(\varphi \cdot M_{-}\right)\right\rangle-\left\langle A u,\left(\varphi \cdot M_{-}\right)\right\rangle,
\end{aligned}
$$

where in the last equality we have used (3.2) with $S=\varphi \cdot M_{-}$. Therefore

$$
\langle u, \check{\varphi}\rangle=\left\langle\left(k_{-}^{-\alpha}+\lambda k_{-}^{-\beta}\right) * u,\left(\varphi \cdot M_{-}\right) \overline{ }\right\rangle-\left\langle A u,\left(\varphi \cdot M_{-}\right) \overline{ }\right\rangle=\left\langle\Delta^{\alpha} u+\lambda \Delta^{\beta} u-A u,\left(\varphi \cdot M_{-}\right)\right\rangle .
$$

We conclude that $\left\langle T_{\alpha, \beta} f, \check{\varphi}\right\rangle=\left\langle f,\left(\varphi \cdot M_{-} \check{)}\right\rangle\right.$ and then $M(t)$ is an $\ell_{p}$-multiplier.

It only remains to prove that $(i i)$ implies $(i)$. We first claim that $N(t):=\left(1-e^{-i t}\right)^{\alpha}\left(\left(1-e^{-i t}\right)^{\alpha}+\lambda\left(1-e^{-i s}\right)^{\beta}-\right.$ $A)^{-1}$ and $S(t)=\left(1-e^{-i t}\right)^{\beta}\left(\left(1-e^{-i t}\right)^{\alpha}+\lambda\left(1-e^{-i t}\right)^{\beta}-A\right)^{-1}$ are $\ell_{p}$-multipliers. Indeed, since $\left.N(t)=f_{\alpha}(t)\right) M(t)$ and $\left.S(t)=f_{\beta}(t)\right) M(t)$ where $f_{\alpha}(t)=\left(1-e^{-i t}\right)^{\alpha}$ and $f_{\beta}(t)=\left(1-e^{-i t}\right)^{\beta}$, the $R$-boundedness of $N(t)$ and $S(t)$ follows. On the other hand, the identities:

$$
\begin{aligned}
\left(1-e^{i t}\right)\left(1+e^{i t}\right) N^{\prime}(t) & =-i \alpha N(t)\left(1+e^{i t}\right)+i \alpha N(t)^{2}\left(1+e^{i t}\right)+i \lambda \beta N(t) S(t)\left(1+e^{i t}\right) \\
\left(1-e^{i t}\right)\left(1+e^{i t}\right) S^{\prime}(t) & =-i \beta S(t)\left(1+e^{i t}\right)+i \alpha S(t) N(t)\left(1+e^{i t}\right)+i \lambda \beta S(t)^{2}\left(1+e^{i t}\right)
\end{aligned}
$$

show that the sets $\left\{\left(1-e^{i t}\right)\left(1+e^{i t}\right) N^{\prime}(t): t \in \mathbb{T}\right\}$ and $\left\{\left(1-e^{i t}\right)\left(1+e^{i t}\right) S^{\prime}(t): t \in \mathbb{T}\right\}$ are $R$-bounded and then the claim holds by Theorem 2.9. Let $f \in \ell_{p}(\mathbb{Z} ; X)$ be given. By hypothesis, there exists $u \in \ell_{p}(\mathbb{Z} ;[D(A)])$ such that

$$
\sum_{n \in \mathbb{Z}} u(n) \check{\varphi}(n)=\sum_{n \in \mathbb{Z}}\left(\varphi \cdot M_{-}\right)(n) f(n)
$$


for all $\varphi \in C_{\text {per }}^{\infty}(\mathbb{T})$. On the other hand, there exist $v, w \in \ell_{p}(\mathbb{Z} ;[D(A)])$ such that

$$
\begin{aligned}
& \sum_{n \in \mathbb{Z}} v(n) \check{\psi}(n)=\sum_{n \in \mathbb{Z}}\left(\psi \cdot N_{-}\right)(n) f(n) \\
& \sum_{n \in \mathbb{Z}} w(n) \check{\eta}(n)=\sum_{n \in \mathbb{Z}}\left(\eta \cdot S_{-} \check{)}(n) f(n)\right.
\end{aligned}
$$

for all $\psi, \eta \in C_{p e r}^{\infty}(\mathbb{T})$. Since $N(t)=\widehat{k}^{-\alpha}(t) M(t)$ we have

$$
\left(\psi \cdot N_{-} \check{)}(n)=\frac{1}{2 \pi} \int_{-\pi}^{\pi} e^{i n t} \psi(t) \widehat{k}^{-\alpha}(-t) M(-t) d t .\right.
$$

Choosing $\varphi(t)=\psi(t) \widehat{k^{-\alpha}}(-t)$ in (3.4) we obtain

$$
\langle v, \check{\psi}\rangle=\left\langle u,\left(\psi \cdot \widehat{k_{-}^{-\alpha}}\right)\right\rangle
$$

and hence by Lemma 2.7 we get that $k^{-\alpha} * u \in \ell_{p}(\mathbb{Z} ; X)$ and

$$
\Delta^{\alpha} u(n)=k^{-\alpha} * u(n)=v(n), \quad n \in \mathbb{Z} .
$$

Analogously, since $S(t)=\widehat{k}^{-\beta}(t) M(t)$ we can choose $\varphi(t)=\eta(t) \widehat{k^{-\beta}}(-t)$ in (3.4) and then, by Lemma 2.7 we get that $k^{-\beta} * u \in \ell_{p}(\mathbb{Z} ; X)$ and

$$
\Delta^{\beta} u(n)=k^{-\beta} * u(n)=v(n), \quad n \in \mathbb{Z} .
$$

Now, from the identity $N(t)+\lambda S(t)=A M(t)+I$ we obtain after multiplication by $e^{i n t} \varphi(t)$ and then integration on the interval $(-\pi, \pi)$, the identity

$$
\left(\varphi \cdot N_{-}\right)(n)+\lambda\left(\varphi \cdot S_{-}\right)(n)=A\left(\varphi \cdot M_{-} \check{)}(n)+\check{\varphi}(n) I,\right.
$$

for all $\varphi \in C_{p e r}^{\infty}(\mathbb{T})$. Then we get

$$
\left\langle f,\left(\varphi \cdot N_{-}\right) \overline{\rangle}+\left\langle f, \lambda\left(\varphi \cdot S_{-} \check{)}\right\rangle=\left\langle f, A\left(\varphi \cdot M_{-} \check{)}\right\rangle+\langle f, \check{\varphi}\rangle .\right.\right.\right.
$$

Replacing (3.4), (3.5) and (3.6) in the above identity we obtain

$$
\sum_{n \in \mathbb{Z}} v(n) \check{\varphi}(n)+\lambda \sum_{n \in \mathbb{Z}} w(n) \check{\varphi}(n)=\sum_{n \in \mathbb{Z}} A u(n) \check{\varphi}(n)+\sum_{n \in \mathbb{Z}} \check{\varphi}(n) f(n),
$$

for all $\varphi \in C_{\text {per }}^{\infty}(\mathbb{T})$. Considering (3.7), (3.8) and replacing $\varphi_{k}(t):=e^{-i k t}, k \in \mathbb{Z}$ we conclude that $u$ satisfies the equation (3.1).

In order to show uniqueness, we consider $u: \mathbb{Z} \rightarrow[D(A)]$ one solution of $(3.1)$ with $f \equiv 0$. For all $\varphi \in C_{\text {per }}^{\infty}(\mathbb{T})$ and using (3.3) we obtain

$$
\langle u, \check{\varphi}\rangle=\left\langle\Delta^{\alpha} u-A u,\left(\varphi \cdot M_{-}\right)^{\check{\gamma}}\right\rangle=0 .
$$

Choosing $\varphi_{k}(t):=e^{-i k t}, k \in \mathbb{Z}$ we obtain $u \equiv 0$. This proves $(i)$ and the theorem.

The following statement follows from the closed graph theorem and Theorem 3.2.

Corollary 3.3. In the context of Theorem 3.2, if condition (iii) is valid, we have $u, \Delta^{\alpha} u, \Delta^{\beta} u, A u \in l_{p}(\mathbb{Z}, X)$. Moreover, there exists a constant $C>0$ such that

$$
\left\|\Delta^{\alpha} u\right\|_{p}+\lambda\left\|\Delta^{\beta} u\right\|_{p}+\|A u\|_{p} \leq C\|f\|_{p} .
$$

As a consequence of Theorem 3.2, we easily have a corresponding one in the case of Hilbert spaces, where $R$-boundedness is equivalent to norm boundedness [18, Chapter 3, Remark 3.2]. 
Corollary 3.4. Let $H$ be a Hilbert space and $\alpha, \beta>0, \lambda \geq 0$. Suppose that $\left\{\left(1-e^{-i t}\right)^{\alpha}+\lambda\left(1-e^{-i t}\right)^{\beta}\right\}_{t \in \mathbb{T}} \subset$ $\rho(A)$. The following assertions are equivalent

(i) For all $f \in \ell_{p}(\mathbb{Z}, H)$ there exists a unique $u \in \ell_{p}(\mathbb{Z}, H)$ such that $u(n) \in D(A)$ for all $n \in \mathbb{Z}$ and $u$ satisfies (3.1);

(ii) $\sup _{t \in \mathbb{T}}\left\|\left(\left(1-e^{-i t}\right)^{\alpha}+\lambda\left(1-e^{-i t}\right)^{\beta}-A\right)^{-1}\right\|<\infty$.

Now, we can consider the nonlinear perturbed version of (3.1) given by

$$
\Delta^{\alpha} u(n)+\lambda \Delta^{\beta} u(n)=A u(n)+G(u)(n)+\rho f(n)
$$

where $\rho>0, f \in \ell_{p}(\mathbb{Z}, X)$ and $G: \ell_{p}(\mathbb{Z}, X) \rightarrow \ell_{p}(\mathbb{Z}, X)$. We can show a result concerning the existence of $\ell_{p}(\mathbb{Z}, X)$-solutions of (3.10) in terms of the symbol of the equation and the regularity of $G$.

Theorem 3.5. Let $X$ be a UMD space, $1<p<\infty, \alpha, \beta>0$ and $\lambda \geq 0$. Suppose that $\left\{\left(1-e^{-i t}\right)^{\alpha}+\lambda(1-\right.$ $\left.\left.e^{-i t}\right)^{\beta}\right\}_{t \in \mathbb{T}} \subset \rho(A)$. If the following conditions hold

(i) the set $\left\{\left(\left(1-e^{-i t}\right)^{\alpha}+\lambda\left(1-e^{-i t}\right)^{\beta}-A\right)^{-1}\right\}_{t \in \mathbb{T}}$ is $R$-bounded,

(ii) $G$ is continuously Fréchet differentiable at $u=0, G(0)=0$ and $G^{\prime}(0)=0$,

then there exists $\rho^{*}$ such the equation (3.10) has a solution $u=u_{\rho} \in \ell_{p}(\mathbb{Z}, X)$ for each $\rho \in\left[0, \rho^{*}\right)$.

Proof. We note that $\|u\|:=\left\|\Delta^{\alpha} u\right\|+\lambda\left\|\Delta^{\beta} u\right\|+\|A u\|+\|u\|$ defines a norm in $\ell_{p}(\mathbb{Z},[D(A)])$ and hence $\left(\ell_{p}(\mathbb{Z},[D(A)]),\|\cdot|\||)\right.$ becomes a Banach space. Let $L: \ell_{p}(\mathbb{Z},[D(A)]) \rightarrow \ell_{p}(\mathbb{Z},[D(A)])$ be defined as $(L u)(n):=$ $\Delta^{\alpha} u(n)+\lambda \Delta^{\beta} u(n)-A u(n)$.

By Corollary 3.4, since hypothesis ( $i$ ) holds, we have that the inequality (3.9) holds, and then we have $\|u\| \leq C\|L u\|$ for some constant $C>0$. Also, by definition of $L$, we have $\|L u\| \leq\|u\|$. Then $L$ defines an isomorphism. Given $\rho \in[0,1)$, we define:

$$
H[u, \rho]=-L u+G(u)+\rho f
$$

By hypothesis $(i i)$, we have $H[0,0]=0$ and $H$ is continuously differentiable at $(0,0)$. In addition, $H_{(0,0)}^{1}=-L$ which is invertible. Therefore, using the implicit function theorem, we deduce the existence of $\rho^{*}$ such that for all $\rho \in\left[0, \rho^{*}\right)$, there exists $u_{\rho} \in \ell_{p}(\mathbb{Z}, X)$ such that $H\left[u_{\rho}, \rho\right]=0$. This proves the theorem.

\section{Examples}

We verify the conditions provided in Theorem 3.5 in order to show the existence and uniqueness of $\ell_{p}(\mathbb{Z}, X)$ solutions for the following equation

$$
\Delta^{\alpha} u(n, x)+\lambda \Delta^{\beta} u(n, x)+b u(n, x)-\frac{\partial^{2}}{\partial x^{2}} u(n, x)=|u(n, x)|^{c} u(n, x)+\varepsilon f(n, x)
$$

where $\lambda \geq 0$ is fixed, $b$ and $c>1$ are real numbers, $\varepsilon>0$ and $f \in \ell_{p}(\mathbb{Z}, X)$ is an external force whose size is controlled by $\epsilon$. Note that the linear part of the equation (4.1) corresponds to the discrete time Telegraph equation when $\alpha=2, \beta=1, b=0, \lambda=\frac{1}{\tau}$ and $A=\frac{\rho}{\tau} \partial_{x x}$. Also, it coincides with the discrete time Klein-Gordon equation for $\alpha=1, \lambda=0$ and $A=\partial_{x x}-b$.

Equation (4.1) can be modeled as (3.10) for $A u=u^{\prime \prime}-b u$ defined on $H^{2}(\mathbb{R})$ and $G(u)=|u|^{c} u$. It is well known that the operator $B u=u^{\prime \prime}$ with domain $D(B)=H_{0}^{2}(\mathbb{R})$ generates a contraction $C_{0}$-semigroup on $L^{2}(\mathbb{R})$, therefore the following estimate for their resolvent operator holds

$$
\left\|(\mu-B)^{-1}\right\| \leq \frac{1}{\Re(\mu)}, \text { for all } \Re(\mu)>0 .
$$


Then, for all $0<\alpha, \beta \leq 2$ we have

$$
\begin{aligned}
\Re\left(\left(1-e^{-i t}\right)^{\alpha}+\lambda\left(1-e^{-i t}\right)^{\beta}\right) & =(2-2 \cos (t))^{\frac{\alpha}{2}} \cos \left(\alpha \arctan \left(\frac{\sin (t)}{1-\cos (t)}\right)\right) \\
& +\lambda(2-2 \cos (t))^{\frac{\beta}{2}} \cos \left(\beta \arctan \left(\frac{\sin (t)}{1-\cos (t)}\right)\right) \\
& >(2-2 \cos (t))^{\frac{\alpha}{2}} \cos \left(\frac{\alpha \pi}{2}\right)+\lambda(2-2 \cos (t))^{\frac{\beta}{2}} \cos \left(\frac{\beta \pi}{2}\right) \\
& >-\left(2^{\alpha}+\lambda 2^{\beta}\right) .
\end{aligned}
$$

As a consequence, for all $b \geq 2^{\alpha}+\lambda 2^{\beta}$ we get that $\left(1-e^{-i t}\right)^{\alpha}+\lambda\left(1-e^{-i t}\right)^{\beta} \in \rho(A)$ and (4.2) shows that

$$
\sup _{t \in \mathbb{T}}\left\|\left(\left(1-e^{-i t}\right)^{\alpha}+\lambda\left(1-e^{-i t}\right)^{\beta}-A\right)^{-1}\right\| \leq \frac{1}{b-\left(2^{\alpha}+\lambda 2^{\beta}\right)}<\infty .
$$

Furthermore, $G$ is a Fréchet differentiable function at $u=0$ and clearly satisfies that $G^{\prime}(0)=0$ since $c>1$. Then, by Theorem 3.5, we conclude that whenever $b>2^{\alpha}+\lambda 2^{\beta}$ there exists a number $\varepsilon^{*}>0$ such that for all $\varepsilon \in\left(0, \varepsilon^{*}\right)$ there exists a solution $u^{\epsilon} \in \ell_{p}(\mathbb{Z}, X)$ of the perturbed fractional damping difference equation (4.1).

Remark 4.1. In particular, this example shows that the discrete Klein-Gordon equation (1.1) admits non-trivial square-summable solutions defined on $\mathbb{Z}$, for small and square-summable external forcing terms whenever $b>2$. In the case of the generalized discrete Basset equation

$$
\Delta^{2} u(n)+\mu \Delta^{\beta} u(n)+b u(n)=f(n), \quad n \in \mathbb{Z}, \quad \mu, b>0, \quad \beta>0,
$$

we obtain that for any $f \in \ell_{p}(\mathbb{Z})$, there exists $p$-summable solutions whenever $b>4$ and $\mu<\frac{b-4}{2^{\beta}}$.

Acknowledgements. C. Lizama has been partially supported by CONICYT - PIA - Anillo ACT1416 and FONDECYT grant 1140258. M. Murillo-Arcila has been supported by MEC, grant MTM2016-75963-P.

[1] L. Abadías and C. Lizama, Almost automorphic mild solutions to fractional partial difference-differential equations. Appl. Anal., 95 (6) (2016), 1347-1369.

[2] L. Abadías, C. Lizama, P.J. Miana and M.P. Velasco. Cesàro sums and algebra homomorphisms of bounded operators. Israel J. Math., 216 (1) (2016), 471-505.

[3] L. Abadías and P.J. Miana. Generalized Cesàro operators on Sobolev-Lebesgue sequence spaces. Preprint.

[4] R. P. Agarwal. Difference Equations and Inequalities. Theory, Methods, and Applications. Second edition. Monographs and Textbooks in Pure and Applied Mathematics, 228. Marcel Dekker, Inc., New York, 2000.

[5] R. P. Agarwal, C. Cuevas and C. Lizama, Regularity of Difference Equations on Banach Spaces, SpringerVerlag, Cham, 2014.

[6] E. Alvarez and C. Lizama. Weighted pseudo almost automorphic and S-asymptotically w-periodic solutions to fractional difference-differential equations. Electronic J. Diff. Equ., 270 (2016), 1-12.

[7] T. M. Atanackovic, S. Pilipovic and D. Zorica. A diffusion wave equation with two fractional derivatives of different order. J. Phys. A: Math. Theor. 40 (2007), 5319-5333.

[8] F.M. Atici and S. Sengul. Modeling with fractional difference equations. J. Math. Anal. Appl., 369 (2010), $1-9$.

[9] F. M. Atici and P. W. Eloe. Initial value problems in discrete fractional calculus. Proc. Amer. Math. Soc., 137 (3), (2009), 981-989.

[10] D. Baleanu, R. Garra, I. Petras. A fractional variational approach to the fractional Basset-type equation. Rep. Math. Phys. 72 (1) (2013), 57-64. 
[11] H. Beyer, S. Kempfle. Definition of physically consistent damping laws with fractional derivatives. Z. Angew. Math. Mech. 75 (8) (1995), 623-635.

[12] H. Bateman. Some simple differential difference equations and the related functions. Bull. Amer. Math. Soc. 49 (1943), 494-512.

[13] S. Blunck. Maximal regularity of discrete and continuous time evolution equations. Studia Math. 146 (2) (2001), 157-176.

[14] R. Dahal and C. S. Goodrich. A monotonicity result for discrete fractional difference operators. Arch. Math. (Basel) 102 (3) (2014), 293-299.

[15] I. K. Dassios. Stability and robustness of singular systems of fractional nabla difference equations. Circuits Syst Signal Process (2016). doi:10.1007/s00034-016-0291-x.

[16] I. K. Dassios and D.I. Baleanu. Duality of singular linear systems of fractional nabla difference equations. Applied Mathematical Modelling, (2014). DOI 10.1016/j.apm.2014.12.039.

[17] I. K. Dassios. Geometric relation between two different types of initial conditions of singular systems of fractional nabla difference equations. Math. Meth. Appl. Sci. DOI: 10.1002/mma.3771.

[18] R. Denk, M. Hieber and J.Prüss. $\mathcal{R}$-boundedness, Fourier multipliers and problems of elliptic and parabolic type. Mem. Amer. Math. Soc. 166 (788) 2003.

[19] S. Elaydi. An Introduction to Difference Equations. Third edition. Undergraduate Texts in Mathematics. Springer-Verlag, New York, 2005.

[20] R. Ferreira. Existence and uniqueness of solution to some discrete fractional boundary value prob-lems of order less than one. J. Differ. Equ. Appl. 19, (2013), 712-718.

[21] A. K.Golmankhaneh, A. Golmankhaneh and D. Baleanu. On nonlinear fractional Klein-Gordon equation. Signal Processing 91 (2011), 446-451.

[22] C. S. Goodrich. Existence of a positive solution to a system of discrete fractional boundary value problems. Appl. Math. Comput. 217 (2011), 4740-4753.

[23] C. S. Goodrich. A convexity result for fractional differences. Appl. Math. Letters 35 (2014), 58-62.

[24] M. T. Holm. The Laplace transform in discrete fractional calculus. Comp. Math. Appl., 62 (3) (2011), $1591-1601$.

[25] B. Jin, B. Li, Z. Zhou. Discrete maximal regularity of time-stepping schemes for fractional evolution equations. arXiv:1606.07587v1 [math.NA].

[26] T. Kemmochi. Discrete maximal regularity for abstract Cauchy problems. Studia Math. 234 (3) (2016), 241-263.

[27] B. Kovács, B. Li and C. Lubich. A-Stable time discretizations preserve maximal parabolic regularity. SIAM J. Numer. Anal. 54 (6) (2016), 36003624.

[28] C. Lizama. $\ell_{p}$-maximal regularity for fractional difference equations on UMD spaces. Math. Nach., 288 (17/18) (2015), 2079-2092.

[29] C. Lizama. The Poisson distribution, abstract fractional difference equations, and stability. Proc. Amer. Math. Soc., to appear.

[30] C. Lizama and M. Murillo-Arcila. $\ell_{p}$-maximal regularity for a class of fractional difference equations on UMD spaces: The case $1<\alpha<2$. Banach J. Math. Anal. 11 (1) (2017), 188-206.

[31] C. Lizama and M. Murillo-Arcila. Well-Posedness for a class of discrete time fractional models. Submitted. 
[32] F. Mainardi. Fractional Calculus, Some Basic Problems in Continuum and Statistical Mechanics, CISM lectures 1997.

[33] M.D. Ortigueira, F.J. V. Coito and J.J. Trujillo. Discrete-time differential systems. Signal Processing. 107 (2015), 198-217.

[34] V. E. Tarasov. Fractional-order difference equations for physical lattices and some applications. J. Math. Phys. 56 (10) (2015), 1-19.

[35] A. Zygmund. Trigonometric series. 2nd ed. Vol. I, II. New York (NY): Cambridge University Press, 1959. 